\title{
Comparative Study Between Two Empirical Antibiotic Regime in the Management of Childhood Malignancy with Fever
}

\author{
L Helaly ${ }^{1}$, M Z H Sarker ${ }^{2}$, M A Mannan ${ }^{3}$, M T Hossain ${ }^{4}$, S Ahmed ${ }^{5}$, M Begum $^{6}$
}

1 Dr.Laila Helaly

Consultant

Bangladesh Thalassaemia

Hospital, Dhaka

${ }^{2}$ Dr. Md Zakir Hossain Sarker Assistant Professor NIDCH

${ }^{3}$ Prof. M.A Mannan

Ex Professor

BSMMU

${ }^{4}$ Dr. Md. Tafazzal Hossain

MO. Sonamuri

Noakhali

${ }^{5}$ Dr. Shafi Ahmed

Assistant Professor

Northern International Medical

Colllege

${ }^{6}$ Dr. Momtaj Begum

Associate Professor NICRH

\section{Correspondence}

Dr.Laila Helaly

Consultant

Bangladesh Thalassaemia

Hospital, Dhaka

\section{Abstract}

Objective : The present prospective randomized clinical trial was carried out to assess whether combined cefepime and amikacin as empirical antibiotic therapy was more effective than combined ceftriaxone and gentamicin in the treatment of febrile neutropenic children with malignant diseases.

Material \& Methods : The study was conducted in the Pediatric Hematology and Oncology unit of BSMMU over a period of 2 years. (From January 2006 to December 2007) Hospitalised pediatric cancer patients who developed febrile neutropenia following chemotherapy or radiotherapy were the study population. A total 64 cases were consecutively included in the study and were randomly assigned to either cefepime \& amikacin group (Group- A) or ceftriaxone \& gentamicin group (Group-B). The Group-A received cefepime 1500 $\mathrm{mg} / \mathrm{m}^{2} /$ dose infused over 15 minutes in two divided doses intravenously(IV) while amikacin was administered as thrice daily dose of $200 \mathrm{mg} / \mathrm{m}^{2} /$ dose. Patients of Group-B received ceftriaxone 1500 $\mathrm{mg} / \mathrm{m}^{2} /$ dose in two divided doses and gentamicin $60 \mathrm{mg} / \mathrm{m}^{2} /$ dose thrice daily IV. The therapy was continued until absolute neutrophil counts reached $>1000$ neutrophils $/ \mathrm{mm}^{3}$. The treatment outcome was considered successful if fever resolves within 4 days and does not recur within 7 days of completion of therapy. Of the 64 patients, 13 cases were excluded from the final analysis.

Results : Bacteria were isolated from culture in only $16.7 \%$ of cases Group-A and $9.5 \%$ of group-B. Patients E. coli was the most common isolate found in blood specimen (37\%). Following intervention, $90 \%$ of cefepime $\&$ amikacin group and $85.6 \%$ of ceftriaxone \& gentamicin group improved absolute neutrophil count to $>1000 / \mathrm{mm}^{3}$ of blood. Persistence of fever after start of study drug and duration of antibiotic therapy were significantly less in the former group than those in later group ( $p=0.049$ and $p=0.004$ respectively). Only 1 patient of group $B$ had recurrence of infection within 7 days of treatment completion. The mean duration of hospital stay was less in the former group 17.97 \pm 2.61 days) than that in the latter group (11.00 \pm 3.42 days) $(p=$ 0.06). Evaluation of final outcome shows that majority $(86.6 \%)$ of cefepime \& amikacin group had successful outcome, while majority of ceftriaxone \& gentamicin group $(81 \%)$ failed to resolve infection with continuation of fever for $>4$ days.

Conclusion : The study concluded that combined cefepime and amikacin is a better option for empirical treatment of fever and neutropenia in children with malignancies than combined ceftriaxone and gentamicin $(p<0.001)$.

Keywords : Empirical antiboltic, Cefepime, Amikacin, febrile neutropenia, Childhood Malignancy

\section{Introduction}

Infectious diseases are major causes of morbidity and mortality in immunocompromised pediatric patients with cancer ${ }^{1}$. Combination therapy with an aminoglycoside plus an anti-pseudomonal â-lactam has commonly been recommended in febrile neutropenia because this approach provides broad- spectrum coverage, bactericidal activity and potential synergistic effects and minimizes the development of resistance during treatment.

Infection is a major threat in paediatric patients suffering from malignancies where neutropenia is secondary to chemotherapy or radiation therapy. But infection is often difficult to document in these patients. Approximately $40 \%$ of these patients never exhibits culture-documented infection, although they improve clinically after treatment with broad spectrum antibiotics, suggesting an occult microbial source as the cause of fever. The empirical administration of combination of antibiotic therapy, the continuation of the therapy until the neutropenia improves and the fever resolves have become a routine treatment approach in febrile neutropenic patients with malignancies ${ }^{1}$.

Organisms causing infections in neutropenic paediatric patients are indistinguishable from those in the adult population. Bacterial organisms that are frequently isolated when fever is present include aerobic gram-positive cocci and bacilli such as coagulase-positive and negative staphylococci, streptococci, enterococcus faecalis/faecium, Corynebacterium spp. and Gram negative bacilli, such as Escherichia coli, Klebsiella spp. This severe risk of bacterial infection, coupled with the insensitivity of diagnostic tests and delays in the identification of pathogens, warrants the immediate empiric administration of broad spectrum antibiotics ${ }^{2}$.

The risk of infection increases 10 -fold with declining neutrophil counts ${ }^{3}$. Rack off and colleagues in the year 1996 found that the risk of bacteremia in pediatric oncology patients was associated with fever greater than $39^{\circ} \mathrm{C}\left(102^{\circ} \mathrm{F}\right)$ and absolute neutrophil count of less than $100 / \mathrm{mm}^{3}$ at the time of presentation with fever and neutropenia.

Ceftriaxone is less costly but because of its rampant use the resistance to this drug is increasing day by day. Cefepime though costly, resistance has not been developed yet and has wide-spectrum of coverage reducing the suffering and hospital stay of the patients. Thus ultimate cost of treatment is reduced. Amikacin has also broader spectrum of activities against bacteria and less ototoxic than gentamicin.

\section{Objectives}

To assess whether cefepime and amikacin as empirical antibiotic therapy was more or as effective as ceftriaxone and gentamicin in the treatment of febrile 
neutropenic children with malignant diseases.

\section{Material \& Methods}

The following methods and materials were used to conduct the study. Patients with following characteristics were eligible for the study

- children ranging from 1-15 years of either sex

- absolute neutrophil count $<1000 / \mathrm{mm}^{3}$ with fever

- need empiric antibiotic treatment for suspected infection.

- temperature $>38^{\circ} \mathrm{C}\left(>101.3^{\circ} \mathrm{F}\right)$ persisting at least for 1 hour

A total of 64 cases were consecutively included in the study.

Patients were excluded from the study when the study drug had to

be changed due to an adverse effect or microbial resistance or another antibiotic was concomitantly used. Therefore such patients were excluded from the final analysis.

A structured data collection form was developed containing all the variables of interest. Data were collected by interview, observation, clinical and laboratory examination of patients.

After initial evaluation and random allocation of the patients into two groups, the treatment was started according to defined protocol. Patients of Group-A received intravenous regimen consisting of Cefepime $1500 \mathrm{mg} / \mathrm{m}^{2} /$ dose infused over 15 minutes in two divided doses intravenously (IV). Amikacin was administered as thrice daily dose of $200 \mathrm{mg} / \mathrm{m}^{2} /$ dose IV. Patients of Group-B were assigned to receive Ceftriaxone and Gentamicin. Ceftriaxone was given as 1500 $\mathrm{mg} / \mathrm{m}^{2} /$ dose in two divided doses and Gentamicin was given as $60 \mathrm{mg} / \mathrm{m}^{2} /$ dose thrice daily IV. Study drug therapy was continued until absolute neutrophil counts reached $>1000$ neutrophils $/ \mathrm{mm}^{3}$

Data were processed and analysed by using SPSS soft ware.

\section{Result}

The selected children with different malignancies were randomly assigned to Group-A (cefepime \& amikacin) and Group-B (ceftriaxone and gentamicin) and treatment was started with a defined protocol (described in earlier chapter). Of the 64 patients, 13 (2in Group-A and 11 in Group-B) cases were excluded because of early discontinuation of drug due to adverse effect, change of antibiotic, concomitant use of another antibiotics and/or resistant organism isolated and death leaving 51 for final analysis (30 in Group-A and 21 in Group-B). The outcomes of the patients were then compared between the two groups. The findings of the study obtained from data analyses are presented below. No significant difference was observed between groups in terms of age and $\operatorname{sex}(p=0.529$ and $p=0.151$ respectively).

Table 1 : Comparison of demographic variables between groups

\begin{tabular}{lccc} 
Age $(\mathrm{yrs})^{\#}$ & \multicolumn{2}{c}{ Group } & P-value \\
& Group-A $(\mathrm{n}=30)$ & Group-B $(\mathrm{n}=21)$ & \\
$<3$ & $5(16.7)$ & $7(33.3)$ & \\
$3-6$ & $12(40.0)$ & $8(38.1)$ & \\
$6-10$ & $7(23.3)$ & $3(14.3)$ & 0.529 \\
10 & $6(20.0)$ & $3(14.3)$ & \\
Mean \pm SD & $5.88 \pm 3.38$ & $4.86 \pm 3.65$ &
\end{tabular}

Sex

Male

$17(56.7)$

16(76.2)

0.151
Figures in the parentheses denote corresponding percentage.

\# Data were analysed using Chi-square $\left(x^{2}\right)$ Test and level of significance was 0.05

Figure 1 : Correlation between neutrophil count and temperature :

Correlation between absolute neutrophil count and temperature demonstrates that the two variables bear an inverse relationship indication that as neutrophil count increases the temperature correspondingly decreases ( $r=\_0.120, p=0.344$ ) (Fig. 4.)

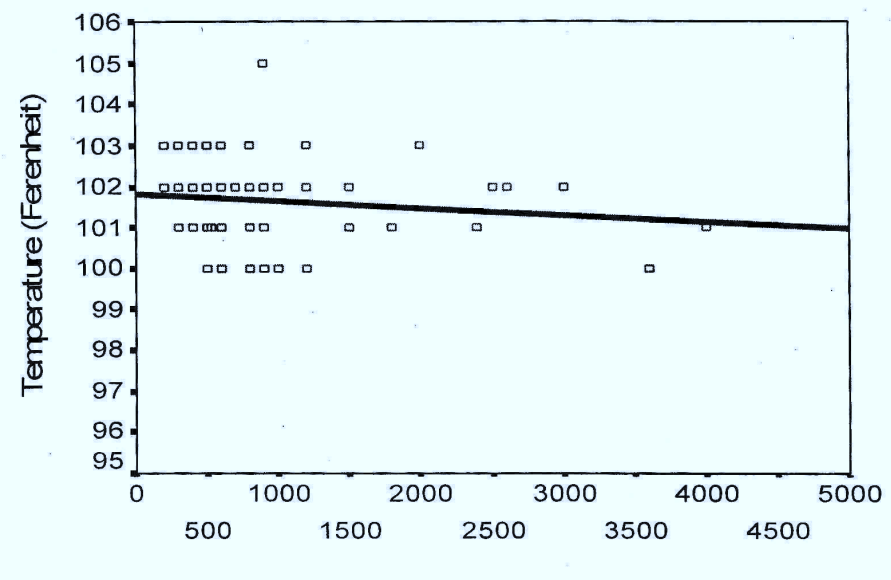

Neutrophil count (mm3)

Fig 2: Correlation between neutrophil count and temperature.

Table II : Comparison of diagnosis of infection between groups.

\begin{tabular}{lccc} 
Diagnosis of infection & \multicolumn{2}{c}{ Group } & P-value \\
& Group-A $(\mathrm{n}=30)$ & Group-B $(\mathrm{n}=21)$ \\
Microbiologically documented & $* 5(16.7)$ & $2 .(9.5)$ & \\
infection (MDI) & & & \\
$\begin{array}{l}\text { Clinically documented infection } \\
\text { (CDI) }\end{array}$ & $7(23.3)$ & $8(38.1)$ & 0.472 \\
Unexplained fever & $18(60.0)$ & $11(52.4)$
\end{tabular}

Figures in the parentheses denote\%; ${ }^{*}$ In Group-A 1 (one) case had two microorganisms. \# Data were analysed using Chi-square $\left(\mathrm{X}^{2}\right)$ Test and level of significance was 0.05 .

Table III: Comparison specimens culture between two groups.

$\begin{array}{cccc}\text { Specimens } & \text { Organisms } & \text { Gp-A } & \text { Gp-B } \\ \text { Blood } & \text { E. Coli } & 3(75) & 0 \\ & \text { Streptococcus } & 1(25) & \\ \text { Urine } & \text { K. Pneumoniae } & 1(100) & 0 \\ \text { Stool } & \text { Shigella } & 0 & 1(100) \\ \text { Pus } & \text { Staphylococcus } & 1(100) & 1(100)\end{array}$




\section{Table IV : Evaluation of outcome of patients in completion therapy:}

Outcome variables

Group

P-value

Neutrophil count \#

$500-1000$

Group-A ( $n=30)$ Group-B $(n=21)$

$>1000$

$$
3(10.0)
$$$$
\text { 27.(90.0) }
$$

$3(14.4)$

$18(85.6)$

Persistence of fever after

$3.57 \pm 1.75$

$6.76 \pm 3.35$

$0.049 \mathrm{~s}$

start of study drug (days)*

During of antibiotic

therapy (days)*

$6.90 \pm 1.42$

00

$9.48 \pm 2.68$

$1(4.8)$

$0.004 \mathrm{~s}$

0.412

Recur of infection within 7 days of treatment completion

Hospital stay (days)*

$7.97 \pm 2.61$

$11.00 \pm 3.42$

Figure in the parentheses denote corresponding percentage; $\mathrm{S}=$ Significant.

* Student t-Test was done to analysed the data; \# Data were analysed Fisher Exact Test;

Table V : Comparison of final outcome between groups $(n=51)$.

Final outcome Group

$P$-value

\begin{tabular}{|c|c|c|}
\hline & Group-A $(n=30)$ & Group-B $(n=21)$ \\
\hline Success & $26(86.7)$ & $4(19.0)$ \\
\hline Failure & $4(13.3)$ & $17(81.0)$ \\
\hline
\end{tabular}

Figure in the parentheses denote corresponding percentage \# Data were analysed using Chi-square $\left(X^{2}\right)$ Test; $S=$ Significant.

\section{Discussion}

The demographic characteristics of the patients revealed that Group-A and Group-B were almost homogeneous in terms of age (5.88 \pm 3.84 vs. $4.86 \pm 3.65 p=0.529$ ) with around $65 \%$ of patients 5 or below 5 years of age (Table I). Rahman (2003) in his study showed that $64.3 \%$ patients fell under 5 years of age ${ }^{4}$. A male preponderance was observed in both the groups (56.7\% of Group-A and $76.2 \%$ of Group-B were male) $(p=0.151)$. Previos study revealed that $22 \%$ had microbiologically documented infection, $20 \%$ had clinically documented, $20 \%$ had possible and $17 \%$ had doubtful infection ${ }^{6}$. Rahman (2003) in his study showed average $22 \%$ had clinically proved infection, $15 \%$ had documented microbiologically proved infection and $63 \%$ had no definite cause of fever ${ }^{4}$. These findings coincided with our findings. In our study majorities of the subjects of Group-A (83.3\%) and Group-B (90.5\%) had no documented infection.(Table II) In only $16.7 \%$ of cases Group-A and $9.5 \%$ of group-B bacteria were isolated from culture. E. coli was the most common isolate found in blood specimen (37\%). Next common isolate was Staphyhcoccus aureus from pus (25\%) Shigella from stool culture $(12.5 \%)$, Streptococcus from blood $(12.5 \%)$ and Klebsiella pneumoniae from urine (12.5\%). There was predominance of gram-negative organism in this study (Table III). The findings of the study were almost consistent with Khatoon et al (1989) who showed that E. coli made up of $57 \%$ of total infections, Staphyhcoccus aureus (17\%) followed by Streptococcus viridians $(10.8 \%)$ and Pseudomonas pyocyaneous $(7.1 \%)^{5}$. Three deaths occurred in ceftriaxone and gentamicin group. One patient of ALL died on day 10 due to septicemia, one patient of $\mathrm{NHL}$ died on day 14 due to septicemia with severe gastroenteritis. The third patient of AML died on day 21 for diarrhoea with hypokalemeia. These patients were excluded from the final analysis.
Following intervention, $90 \%$ of cefepime \& amikacin group and $85.6 \%$ of ceftriaxone \& gentamicin group improved absolute neutrophil count to $>1000 / \mathrm{mm}^{3}$ of blood. Persistence of fever after start of study drug and duration of antibiotic therapy were significantly less in the former group than those in latter group ( $p=0.049$ and $p=0.004$ respectively). Only 1 patient in Group-B relapsed within 7 days of treatment completion. The mean duration of hospital stay was less in the former group (7.97 \pm 2.61 days) as opposed to that in the latter group (11.00 \pm 3.42 days) (Table IV).

Sanz et al (2002) conducted a large-scale study and drew conclusion that combined cefepime and amikacin is effective for the empirical treatment of fever in patients with haematological malignancies and severe neutropenia, Success rates were slightly lower (40\%) for patients with MDI. However, in Sanz et al's study (2002), the use of multistep antiinfective strategy led to an overall $96 \%$ clinical success rate which is fairly comparable with success rate of the present study $(86.6 \%)^{7}$

In our study, evaluation of final outcome shows that majority (86.6\%) of cefepime \& amikacin group had successful outcome, while majority of ceftriaxone \& gentamicin group (81\%) failed to resolve infection with continuation of fever for $>4$ days (Table-v). We therefore agree with Sanz et al (2002) that combined cefepime and amikacin is better option for empirical treatment of fever and neutropenia in children with malignancies than combined ceftriaxone and gentamicin $(p<0.001)^{7}$.

\section{Conclusion}

From the finding of the present study and discussion thereof, it can be concluded that combined cefepime and amikacin is a better option for empirical treatment of fever and neutropenia in children with malignancies than combined ceftriaxone and gentamicin $(p<0.001)$. However, the sample size was small and the patients were not followed up for longer period. A further study, therefore, should be conducted with large sample size.

\section{References}

1. Walsh, TJ, Roilides, E, Groll, AH, Gonzalez C, Pizzo, PA, 2003, 'Infectious complications in pediatric cancer patients', In: Principles and Practice of Pediatric Oncology, Philadelphia, Williams \& Wilkins.

2. Mustafa, MM, Carlson, L, Tkaczewski, I, McCraken, GH and Buchanan, GR 2001, 'Comparative Study of cefepime versus ceftazidime in the empiric_treatment of pediatric cancer patients with fever and neutropenia', Pediatr Dis J, vol. 20, pp. 362-9.

3. Hughes, WT, Armstrong, D \& Bodey, GP et al. 1997, 'Guidelines for the use of antimicrobial agents in neutropenic patients with unexplained fever', Clin Infec Dis, vol.25, pp.551-73.

4. Rahman, A 2003, 'Outcome of two empirical antibiotic regime in the management of childhood malignancy with fever, MD Thesis, Pediatric Haematology \& Oncology, BSMMU.

5. Khatoon, S, Mannan, ${ }^{1}$ MA and Khan, MR 1989, 'Fever in malignant disease of childhood: L- A clinico-pathological study", Bangladesh J Child Health, vol. 13, pp. 16-24. Klaassen, R, Goodman, TR, Pham, B and Doyle, J 2000, 'Low risk prediction rule for pediatric oncology patients presenting with fever and neutropenia', J Clin Oncol, vol.18, pp. 1012-9.

6. The EORTC International Antimicrobial Therapy Project Group 1978, 'Three antibiotic regimens in the treatment of infection in febrile granulocytgopenic patients with cancer' $\mathrm{J}$ Injec Dis, P. 137.

7. Sanz, MA, Lopez, J, Lahuerta, JJ, Rovira, M, Batlle, M and Perez, C 2000, 'Cefepime plus amikacin versus piperacillin- tazobactam plus amikacin for initial antibiotic therapy in hematology patients with febrile neutropenia: results of an open, randomized, multicentre trial', J. Antmicrobial Chemotherapy; vol. 50, pp. 79 - 88. 